American Journal of Infectious Diseases 4 (4): 209-214, 2008

ISSN 1553-6203

(C) 2008 Science Publications

\title{
Low-Birth Weight and Pre-Term Delivery in Relation to Lopinavir/Ritonavir Use in Pregnancy
}

\author{
${ }^{1,2}$ Jorge Senise, ${ }^{1}$ Reisson Cruz, ${ }^{1}$ Ricardo Palacios, ${ }^{1,2}$ Simone Bonafé, \\ ${ }^{1}$ Maria Jose Rodrigues Vaz, ${ }^{3}$ Ana Paula Lacerda, \\ ${ }^{1}$ Abes Ahmed and ${ }^{1}$ Adauto Castelo \\ ${ }^{1}$ Multidisciplinary Group for Infectious Diseases on Pregnancy-NUPAIG, \\ Hospital Sao Paulo, UNIFESP, São Paulo, Brazil \\ ${ }^{2}$ Department of Infectious Diseases, Hospital Ipiranga, São Paulo, Brazil \\ ${ }^{3}$ Abbott Laboratórios do Brasil, São Paulo, Brazil
}

\begin{abstract}
The toxic potential of nevirapine in pregnant women with CD4 count over 250 cells $\mathrm{mm}^{-3}$ and the unsatisfactory efficacy of nelfinavir in patients with baseline Viral Load (VL) over 100,000 copies $\mathrm{mL}^{-1}$ has prompted the use of Lopinavir/ritonavir (LPV/r) in selected situations. This study aims to assess safety of LPV/r in pregnancy. Medical records from pregnant women receiving LPV/r were retrospectively reviewed. Charts corresponding to twin pregnancy, hypertension and having a lack of data supporting a reliable estimate of Gestational Age (GA) at delivery were excluded. Low Birth Weight (LBW) was defined as less than $2500 \mathrm{~g}$. Pre-Term Delivery (PD), defined as GA at delivery less than 259 days, was estimated using date of Last Menstruation Period (LMP) and obstetrical ultrasound. A total of 64 women were analyzed. LPV/r was used in $46.9 \%$ due to virologic failure with other Protease Inhibitors (PIs). LPV/r was used for a mean of 108.8 days. Baseline median CD4+ cell count and HIV-1 RNA were $287 \mathrm{~mm}^{-3}$ and 31,100 copies $\mathrm{mL}^{-1}$, respectively and $345 \mathrm{~mm}^{-3}$ and less than 400 copies $\mathrm{mL}^{-1}$ at delivery. HIV-1 was not transmitted to any newborn. LBW was observed in $13(20.3 \%)$ and PD in $16(25 \%)$ newborns. Time on LPV/r during pregnancy, maternal age, baseline CD4+ cell count and HIV-1 RNA, GA at initiation of LPV/r, reason for prescribing $\mathrm{LPV} / \mathrm{r}$ and type of delivery were not associated with PD. Frequencies of LBW and PD were, respectively, 20.3 and $25 \%$. Neither the magnitude nor the timing in pregnancy of LPV/r use was associated with PD.
\end{abstract}

Key words: HIV mother-to-child transmission, lopinavir, pregnancy, pre-term delivery, low-birth weight

\section{INTRODUCTION}

In countries where antiretrovirals (ARVs) are widely available, the optimal ARV regimen for prevention of mother-to-child transmission during pregnancy is currently under debate. Until recently, most guidelines recommended either nevirapine or nelfinavir in combination with two nucleoside reverse transcriptase inhibitors (NRTIs), usually zidovudine (AZT)+lamivudine (3TC) in HIV-infected pregnant women who do not have evidence of drugresistant virus. However, the use of both nevirapine and nelfinavir has been questioned because of either an increased risk for toxicity or subtherapeutic plasma levels, respectively ${ }^{[1,2]}$.
The development of severe nevirapine-associated skin rash has been reported to be 5.5-7.3 times more common in women than men and has been reported in pregnant women ${ }^{[3,4,5]}$. The degree of risk for hepatic toxicity varies with CD4+ cell count. Women with CD4+ cell counts greater than 250 cells $\mathrm{mm}^{-3}$ who initiate nevirapine including pregnant women receiving ARV drugs solely for prevention of transmission, have an increased risk of developing symptomatic, often rash-associated, nevirapine-related hepatotoxicity, which can be severe, life-threatening and in some cases fatal ${ }^{[6,7]}$. Nevirapine, as a component of an ARV regimen during pregnancy, should therefore either be avoided or used with great caution ${ }^{[8]}$. Additionally, the potency of nelfinavir in cases with elevated HIV-1

Corresponding Author: Adauto Castelo, Multidisciplinary Group for Infectious Diseases on Pregnancy-NUPAIG, Hospital Sao Paulo, UNIFESP, São Paulo, Brazil 
RNA load or previous protease inhibitor (PI) experience with has been shown to be inferior to lopinavir/ritonavir $(\mathrm{LPV} / \mathrm{r})^{[9]}$. In fact, $\mathrm{LPV} / \mathrm{r}$ could be an alternative, both as first-line therapy and for treatment of women with some degree of PI resistance. There are, however, no adequate and well-controlled studies in pregnant women. Furthermore, preliminary studies suggest that an increased LPV/r dose may be required during pregnancy, although specific dosing recommendations have not been established ${ }^{[10]}$. In terms of safety, LPV/r is identified by the Food and Drug Administration (FDA) as pregnancy category $\mathrm{C}$, as are $\mathrm{AZT}$ and $3 \mathrm{TC}^{[11]}$. No treatment-related malformations were observed when LPV/r was administered to pregnant rats or rabbits.

The International AIDS Society-USA Panel and EuroGuidelines Group for HIV-1 Resistance recommend that all pregnant women with detectable HIV-1 RNA levels undergo resistance testing, even if they are ARV naïve, in an effort to maximize the response to ARV drugs in pregnancy ${ }^{[12,13]}$. However, resistance tests are often not readily available and there are situations in pregnancy where therapeutic decisions ought to be taken without delay. The uncertain HIV resistance pattern may make LPV/r a safe therapeutic option, especially for patients who report previous failure/intolerance to nelfinavir or have very high HIV RNA loads.

The objective of this study was to assess the impact of LPV/r, as part of combination therapy prescribed to treat HIV-infected pregnant women, on the rates of infant Low Birth Weight (LBW) and Pre-Term Delivery (PD), HIV RNA load reduction and ultimately, mother-to-child HIV transmission.

\section{MATERIALS AND METHODS}

A retrospective, descriptive chart review was used to analyze HIV-infected pregnant women who received $\mathrm{LPV} / \mathrm{r}$ during antenatal care at a specialized outpatient service of UNIFESP Medical School in Sao Paulo, Brazil, from March 2002 through June 2005. LPV/r was prescribed for one of the following reasons:

- Initiation of ARV therapy after 28 weeks of gestation

- HIV RNA greater than 100,000 copies $\mathrm{mL}^{-1}$

- Previous virologic failure in response to other PIs

- Use of LPV/r before current pregnancy

- Intolerance to nelfinavir
PD, defined as Gestational Age (GA) at delivery of less than 259 days, was calculated based on the date of Last Menstruation Period (LMP) and obstetrical ultrasound (US), according to the following algorithm:

- When estimation of GA based on both LMP and US was above or below 259 days, the case was categorized accordingly. The US device was a Toshiba PowerVision 8000 (Toshiba America Medical Systems, Inc., Tustin, CA, USA)

- In the case of disagreement between US and LMP, the US estimation was used if performed before 21 weeks of gestation. On the other hand, because of progressive loss of accuracy of US-estimated Gestational Age (GA) performed after 21 weeks of gestation, LMP was used only if were deemed reliable by the attending obstetrician and if it were concordant with the Ballard estimation based on newborn parameters. Otherwise, the case was excluded from the analysis

Patients were excluded in cases of twin pregnancy, severe hypertension or a lack of data supporting a reliable estimate of GA at delivery. The study was approved by the ethics committee of the UNIFESP Medical School.

\section{RESULTS}

In the study period, 390 HIV-infected women received prenatal care at UNIFESP Medical School. Of those, $74(19 \%)$ were given LPV/r and included in the study. Ten women were excluded from the analysis: one refused to take any drug during gestation, three had twins and six could not be given an accurate GA estimation because they began care late in pregnancy and were not able to recall the LMP reliably. The remaining 64 women received LPV/r at some point during pregnancy for one of the following reasons: $9(14.1 \%)$ were already on $\mathrm{LPV} / \mathrm{r}$ at the time of conception, $14(21.9 \%)$ initiated prenatal care after 28 weeks of gestation, $30(46.9 \%)$ had previous virologic failure with other PIs, six (9.3\%) had baseline HIV RNA over 100,000 copies $\mathrm{mL}^{-1}$ and five $(7.8 \%)$ showed intolerance to nelfinavir during pregnancy.

Mean age was 29.4 years (range 16-41). Sixteen women $(25 \%)$ learned about their HIV status during current pregnancy and $48(75 \%)$ had this diagnosis for an average of 3.7 years (range, 0-13.8 years). The women started prenatal care with a mean GA of 19 weeks (range, 5.8-36.6 weeks). At first appointment, median CD4+ cell count was $289 \mathrm{~mm}^{-3}$ 
(range, 13-811 $\mathrm{mm}^{-3}$ ) and median HIV-1 RNA load was 19,050 copies $\mathrm{mL}^{-1}$ (range, 0 to $>750,000$ copies $\mathrm{mL}^{-1}$ ). A total of $30.2 \%$ had CD4+ counts less than $200 \mathrm{~mm}^{-3}$.

Thirteen women started LPV/r before 14 weeks of gestation: seven were already on LPV/r at the time of conception. One woman discontinued LPV/r at the eighth week of gestation and resumed after 27 weeks and the remaining six stayed on LPV/r throughout gestation. Twenty-four women started LPV/r between 14 and 28 weeks and 27 women started after 28 weeks. $\mathrm{LPV} / \mathrm{r}$ was used for a mean of 106 days, median of 77.5 days and range of 10-282 days.

HIV-1 RNA load decreased from a median of 31,100 copies $\mathrm{mL}^{-1}$ (interquartile range, $4,595-96,000 \mathrm{~mm}^{-3}$ ) at initiation of LPV/r to less than 400 copies $\mathrm{mL}^{-1}$ at delivery in $46(80.7 \%)$ out of the 57 women who started LPV/r during pregnancy. In parallel, median CD4+ cell count went from 287 cells $\mathrm{mm}^{-3}$ (range, $13-811 \mathrm{~mm}^{-3}$ ) before $\mathrm{LPV} / \mathrm{r}$ to 345 cells $\mathrm{mm}^{-3}$ (range, 32-992 $\mathrm{mm}^{-3}$ ).

The NRTI and non-nucleoside reverse transcriptase inhibitors (NNRTI) backbones used together with $\mathrm{LPV} / \mathrm{r}$ are listed in Table 1 . The overriding majority used AZT+3TC (64.6\%). Pre-term delivery did not vary according to the NRTI backbone $(\mathrm{p}=0.25)$.

Elective C-section was performed in 43 (67.2\%) women, emergent $\mathrm{C}$-section in $14(21.9 \%)$ and normal delivery in $7(10.9 \%)$. Of the 14 cases with emergent C-section, 12 had PD. In four cases C-section was indicated due to fetal distress, in eight cases due to premature labor, one case due to membrane rupture and one functional dystocia. All but one woman received intrapartum AZT. There was no case of HIV-1 motherto-child transmission or of birth defects.
Out of the 64 babies, 29 were male. Overall mean birth weight was $2,774.5 \mathrm{~g}$; the median was $2,800 \mathrm{~g}$ (range, 1,795-3,890 g). Low birth weight, defined as less than 2,500 $\mathrm{g}$ but higher than $1,500 \mathrm{~g}$ at birth, was observed in $13(20.3 \%)$ infants. No case of very low birth weight, defined as less than $1,500 \mathrm{~g}$, was diagnosed.

There were $16(25 \%)$ premature newborns. None of them was extremely premature, defined as GA at delivery of less than 30 weeks. In $14(87.5 \%)$ of the premature cases, GA at delivery based on both LMP and US supported the diagnosis of PD. In the remaining two (cases 13 and 15 of Table 2), GA at delivery was lower than 37 weeks only according to the US estimation. Table 2 provides further detail of the 16 premature cases. GA at delivery of these cases ranged from 218 days (31.1 weeks) to 258 days (36.9 weeks); $6(37.5 \%)$ of the premature infants were delivered between 36 and 37 weeks of gestation.

Time on LPV/r during pregnancy was similar between premature (103.6 days) and non-premature $(106.8$ days $)$ cases $(p=0.88)$. Maternal age, baseline CD4+ cell count and HIV-1 RNA load, GA at initiation of LPV/r, calendar year of antenatal care, time of awareness of HIV infection.

Table 1: Nucleoside and non-nucleoside analogs backbones accompanying LPV/r

\begin{tabular}{lcl}
\hline Antiretroviral & Frequency & Cumulative percent \\
\hline AZT+3TC & 42 & 64.6 \\
AZT+3TC+ddI & 11 & 81.5 \\
AZT+3TC+TDF & 1 & 83.1 \\
AZT+ddI & 2 & 86.2 \\
d4T+3TC & 1 & 87.7 \\
d4T+ddI & 4 & 93.8 \\
NRTI+NEV & 4 & 100.0 \\
Total & 65 & \\
\hline
\end{tabular}

Table 2: Gestational age at delivery of the 16 premature onset of labor cases among women who used LPV/r during pregnancy

\begin{tabular}{lllcl}
\hline Patient & $\begin{array}{l}\text { Gestational age based } \\
\text { on US (days) }\end{array}$ & $\begin{array}{l}\text { Gestational age based } \\
\text { on LMP (days) }\end{array}$ & $\begin{array}{l}\text { Gestational age at which } \\
\text { US was performed (weeks) }\end{array}$ & $\begin{array}{l}\text { Gestational age used in } \\
\text { analysis (days) }\end{array}$ \\
\hline 1 & 218 & 221 & 11 & 218 \\
2 & 229 & 231 & 30 & 231 \\
3 & 234 & 233 & 9 & 234 \\
4 & 234 & 238 & 11 & 234 \\
5 & 236 & 239 & 6 & 236 \\
6 & 242 & 230 & 20 & 242 \\
7 & 248 & 247 & 23 & 247 \\
8 & 249 & 255 & 9 & 250 \\
9 & 250 & 241 & 12 & 241 \\
10 & 252 & 256 & 23 & 256 \\
11 & 254 & 254 & 23 & 254 \\
12 & 254 & 260 & 16 & 256 \\
13 & 256 & 256 & 13 & 256 \\
14 & 256 & 260 & 9 & 256 \\
15 & 256 & 254 & 21 & 254 \\
16 & 258 & & 7 & \\
\hline
\end{tabular}


The reason for prescribing LPV/r and type of delivery were not associated with pre-term delivery. Patients who began using ARVs before the current gestation had a higher PD rate $(30.2 \%)$ than those who began during current gestation (14.3\%), but this difference did not reach statistical significance $(p=0.14)$. PD rate was higher among women who underwent emergent $\mathrm{C}$-section $(85.7 \%)$ than those who underwent elective C-section (2.3\%) $(\mathrm{p}<0.0001)$.

\section{DISCUSSION}

In this group of women with fairly advanced HIV-1 infection who received LPV/r during pregnancy, there was no case of HIV-1 mother-to-child transmission. Furthermore, peripartum HIV-1 RNA load was less than 400 copies $\mathrm{mL}^{-1}$ in number $(82.8 \%)$ of the pregnant women using LPV/r. Low birth weight was present in $13(20.3 \%)$ cases, whereas the overall $\mathrm{PD}$ rate was $25 \%$.

As this was not a randomized controlled study, pregnant women who received LPV/r were likely not to be typical of the overall population of HIV-1-infected pregnant women. In fact, all of the eligibility criteria used in this study were associated with unfavorable pregnancy outcomes. An HIV-1 RNA load higher than 100,000 copies $\mathrm{mL}^{-1}$ has been shown to be strongly associated with HIV-1 mother-to-child transmission ${ }^{[14,15,16]}$. Additionally, patients with such elevated HIV-1 RNA loads have been shown to be less responsive to nelfinavir than to $\mathrm{LPV} / \mathrm{r}^{[17]}$ or $\mathrm{TMC}$ $114^{[18]}$. In fact, despite an initial HIV RNA load of 96,000 copies $\mathrm{mL}^{-1}$ (in the 75 th percentile) in the group of pregnant women who initiated LPV/r during pregnancy, $80.7 \%(46 / 57)$ were undetectable at delivery and $91.2 \%(52 / 57)$ had fewer than 1,000 copies $\mathrm{mL}^{-1}$.

Of note, one patient who received only 21 days of $\mathrm{LPV} / \mathrm{r}$ starting at 29 weeks and 5 days went from an initial HIV-1 RNA load of 98,600-513 copies $\mathrm{mL}^{-1}$ at delivery. The use of a potent regimen in this risky condition may have contributed to the lack of HIV-1 mother-to-child transmission.

Fourteen $(21.9 \%)$ women began prenatal care after 28 weeks of gestation, leading to delayed access to HIV testing. Unfavorable pregnancy outcomes are seen more frequently in the United States (USA) than in Europe partly because the USA has a higher number of HIV-infected women coming from socially disadvantaged groups, with poor access to medical care, which leads to higher rates of $\mathrm{PD}^{[19]}$.
Low birth weight was reported in 13 (20.3\%) infants. PD, present in $11(84.6 \%)$ of these infants, was the only variable significantly associated with LBW. The observed rate is similar to what has been reported with the use of combination therapy with nelfinavir ${ }^{[20]}$. Although previous studies associate the presence of very low birth weight with the use of PIs during pregnancy $^{[20,21]}$, no such case was found in this study.

In this study, PD was defined rather judiciously. US, LMP and Ballard estimations of GA were used in accordance with a predefined algorithm. The definition of PD is very precise (less than 259 days), but GA ascertainment is far less precise. An US performed before 13 weeks and 6 days, the most precise period, has a range of error of \pm 7 days surrounding the estimation of GA. Between 14 and 21 weeks of gestation, the range of error surrounding the estimation of GA is \pm 13 days and \pm 21 days or more after 21 weeks. On the other hand, women with lower levels of education may have more difficulty remembering the date of LMP.

Indeed, six women were excluded from the analysis because no ultrasound was available before 21 weeks and the LMP date was not considered reliable. Therefore, the $25 \% \mathrm{PD}$ rate reported herein is likely to be overestimated. Furthermore, other risk factors for PD were present in this cohort of women. Cigarette smoking during pregnancy was reported by $24(37.5 \%)$ of the women, alcohol use by $3(4.7 \%)$ and the use of illicit substances by $6(9.4 \%)$. Prior PD was reported by $7(10.9 \%)$ of the women. However, none of these variables were significantly associated with PD. Exposure to LPV/r during the first trimester of gestation was not associated with a higher risk of PD when compared to exposure during second or third trimester $(\mathrm{p}=0.84)$. This finding has been corroborated by other studies $^{[22]}$.

In a large cohort of HIV-infected women who received ART during pregnancy ${ }^{[23]}$, only combination therapy with a PI (LPV/r not included) was associated with an increased risk of PD. Similarly, the combined results of the European Collaborative Study and the Swiss Cohort Study with pregnant women exposed to ART with a PI also indicated a higher risk (OR: 2.6) of $\mathrm{PD}^{[24]}$. In contrast, in another large study carried out in the United States, after adjusting for covariates, the risk of pre-term and extreme pre-term delivery among women who received combination therapy with a PI, compared to those who received combination therapy without a PI, was not elevated ${ }^{[20]}$. The regular dose of $\mathrm{LPV} / \mathrm{r}$ (400 mg/100 mg) was used in this cohort. 
There have been suggestions, based on a small pharmacokinetic study ${ }^{[25]}$, that during pregnancy, a higher LPV/r dose $(533 \mathrm{mg} / 133 \mathrm{mg})$ should be used in second and third trimesters, especially for women who are PI experienced, in order to reach adequate LPV exposure. LPV AUC was low with standard dosing $(400 \mathrm{mg} / 100 \mathrm{mg}$ ) during the second trimester. However, there is no clear-cut evidence of the clinical benefit of increasing the dose. Furthermore, the safety profile of the regular dose of LPV/r in pregnancy cannot be extrapolated to higher doses.

In conclusion, the use of LPV/r during pregnancy proved safe and was well tolerated. Considering the difficulty associated with treating the cohort of pregnant women studied, the virologic response was very satisfactory. Larger studies to confirm the findings are warranted.

\section{ACKNOWLEDGEMENT}

The authors thank Drs. Luciana Lunardi, Patrícia Fernandes, Sandra Guaré, Pricilla Chow and all the staff from the Department of Obstetrics and the Division of Infectious Diseases of the UNIFESPHospital São Paulo who care of the HIV-infected pregnant women for their valuable inputs to this study.

This study was sponsored by an unrestricted grant from Abbot Laboratórios do Brasil Ltda. Ricardo Palacios was supported by a studentship grant from the Brazilian National Counsel of Technological and Scientific Development-CNPq.

\section{REFERENCES}

1. Wit, F., J. Nellen and A. Bergshoeff et al., 2003. Plasma nelfinavir concentrations are significantly lower in pregnancy. Antivir. Ther., 8: S214-S215.

2. Watts, D.H., R. Balasubramanian and R.T. Maupin Jr. et al., 2004. Maternal toxicity and pregnancy complications in human immunodeficiency virus-infected women receiving antiretroviral therapy: PACTG 316. Am. J. Obstet. Gynecol., 190: 506-516.

3. Patel, S.M., S. Johnson and S.M. Belknap et al., 2004. Serious adverse cutaneous and hepatic toxicities associated with nevirapine use by nonHIV-infected individuals. J. Acquir. Immune. Defic. Syndr., 35 (2): 120-125.

4. Mazhude, C., S. Jones and S. Murad et al., 2002. Female sex but not ethnicity is a strong predictor of non-nucleoside reverse transcriptase inhibitorinduced rash. AIDS, 16 (11): 1566-1568.
5. Knudtson, E., M. Para, H. Boswell, Fan-Havard and P. Drug Rash, 2003. Eosinophilia and systemic symptoms syndrome and renal toxicity with a nevirapine-containing regimen in a pregnant patient with human immunodeficiency virus. Obstet. Gynecol., 101 (5 Pt 2): 1094-1097.

6. Stern, J.O., P.A. Robinson and J. Love et al., 2003. A comprehensive hepatic safety analysis of nevirapine in different populations of HIV infected patients. J. Acquir. Immune. Defic. Syndr., 34 (Suppl 1): S21-S33.

7. Boehringer-Ingelheim, 2005. Pharmaceuticals Inc. Viramune Drug Label.

8. Hitti, J., L.M. Frenkel and A.M. Stek et al., 2004. For the PACTG 1022 Study Team. Maternal toxicity with continuous nevirapine in pregnancy: results from PACTG 1022. J. Acquir. Immune. Defic. Syndr., 36 (3): 772-776.

9. King, M.S., B.M. Bernstein and S.L. Walmsley et al., 2004. Baseline HIV-1 RNA level and CD4 cell count predict time to loss of virologic response to nelfinavir, but not lopinavir/ritonavir, in antiretroviral therapy-naive patients. JID, 190: 280-284.

10. Stek, A., M. Mirochnick and E. Capparelli et al., 2004. Reduced lopinavir exposure during pregnancy: preliminary pharmacokinetic results from PACTG 1026. Program and abstracts of the 15th International AIDS Conference; Bangkok, Thailand. Abstract LbOrB08.

11. Public Health Service Task Force, 2005. Recommendations for Use of Antiretroviral Drugs in Pregnant HIV-1-Infected Women for Maternal Health and Interventions to Reduce Perinatal HIV1 Transmission in the United States, $<$ http://AIDSinfo.nih.gov>.

12. Hirsch, M.S., F. Brun-Vezinet and R.T. D'Aquila et al., 2000. Antiretroviral drug resistance testing in adult HIV-1 infection: recommendations of an International AIDS Society-USA Panel. JAMA, 283 (18): 2417-2426.

13. The EuroGuidelines Group for HIV Resistance, 2001. Clinical and laboratory guidelines for the use of HIV-1 drug resistance testing as part of treatment management: Recommendations for the European setting. AIDS, 15 (3): 309-320.

14. Garcia, P.M., L.A. kalish and J. Pitt et al., 1999. Maternal levels of plasma human immunodeficiency virus type 1 RNA and the risk of perinatal transmission. Women and Infants Transmission Study Group. N. Engl. J. Med., 341 (6): 394-402. 
15. Katzenstein, D.A., M. Mbizvo and L. Zijenah et al., 1999. Serum level of maternal human immunodeficiency virus (HIV) RNA, infant mortality and vertical transmission of HIV in Zimbabwe. J. Infect. Dis., 179 (6): 1382-1387.

16. Cooper, E.R., M. Charurat and L. Mofenson et al., 2002. Combination antiretroviral strategies for the treatment of pregnant HIV-1-infected women and prevention of perinatal HIV-1 transmission. J. Acquir. Immun. Defic. Syndr., 29 (5): 484-494.

17. Walmsley, S., B. Bernstein and M. King et al., 2002. M98-863 Study Team. Lopinavir-ritonavir versus nelfinavir for the initial treatment of HIV infection. N. Engl. J. Med., 346 (26): 2039-2046.

18. Katlama, C., M.T. Carvalho and D. Cooper et al., 2007. TMC114/r outperforms investigator-selected $\mathrm{PI}(\mathrm{s})$ in 3-class-experienced patients: week 24 primary analysis of POWER 1 (TMC114-C213). Program and abstracts of the 3rd International AIDS Society Conference; Rio de Janeiro, Brazil. Abstract WeOaLB0102.

19. Martin, J.A., B.E. Hamilton and S.J. Ventura et al., 2002. Births: final data for 2000. National vital statistics reports. Hyattsville, Md.: National Center for Health Statistics, Vol. 50. No. 5.
20. Tuomala, R.E. and D.E. Shapiro et al., 2002. Antiretroviral therapy during pregnancy and the risk of an adverse outcome. N. Engl. J. Med., 346 (24): 1863-1870.

21. Beckerman, K.C.D. and P. Garcia et al., 2004. Association between Antiretroviral Therapy during pregnancy and prematurity/low birth weight. 11th Conference of Retroviruses and Opportunistic Infection. San Francisco, abstract 97.

22. Kowalska, A. and T. Niemiec et al., 2003. Effect of antiretroviral therapy on pregnancy outcome in HIV-1 positive women. Med. Wieku. Rozwoj., 7 (4 Pt 1): 459-468.

23. Cotter, A.M., A.G. Garcia and M.L. Duthely et al., 2006. Is antiretroviral therapy during pregnancy associated with an increased risk of preterm delivery, low birth weight or stillbirth? J. Infect. Dis., 193 (9): 1195-1201.

24. The European Collaborative Study and the Swiss Mother+Child HIV Cohort Study, 2000. Combination antiretroviral therapy and duration of pregnancy. AIDS, 14: 2913-2920.

25. Mirochnick, M., A. Stek and A. Capparelli et al., 2006. PACTG 1026s Protocol Team. Adequate Lopinavir Exposure Achieved with a Higher Dose during the Third Trimester of Pregnancy. 13th on Retroviruses and Opportunistic Infections. Denver., abstract 710 . 